\title{
Occupational exposure to hydrocarbons and chronic pancreatitis: a case-referent study
}

\author{
R McNamee, J M Braganza, J Hogg, I Leck, P Rose, N M Cherry
}

\begin{abstract}
Objectives-To investigate the hypothesis that hydrocarbon exposure is a risk factor for chronic pancreatitis.

Methods-102 cases of chronic pancreatitis and 204 age and sex matched referents were interviewed about their occupational histories, alcohol and cigarette consumption, and diet. Exposure to hydrocarbons was inferred from interview responses by four assessors who were blind to disease state, and these data were then summarised by a cumulative hydrocarbon exposure (CHE) score. Results-After adjustment for alcohol, cigarettes, dietary antioxidants, and social class, odds ratios for low CHE scores were $1 \cdot 20(90 \% \mathrm{CI}$ : $0 \cdot 62-2 \cdot 35)$ and $2 \cdot 67(90 \%$ CI: $1 \cdot 22-5 \cdot 87)$ for high scores. A test for trend with level of exposure among only those who had exposure scores $>0$ gave $p=0 \cdot 09$. Analysis by type of hydrocarbon was limited to four exposures for each of which there were at least 20 exposed patients. The adjusted $O R$ for paint solvents (any level) was $0.96(90 \%$ CI: 0.48-1.93); for diesel exhaust fumes OR $=2.66(90 \%$ CI: $1 \cdot 05-6 \cdot 73)$; for petrochemicals OR $=1.82(90 \%$ CI: $0 \cdot 80-4 \cdot 11)$; and for chlorinated solvents $O R=1.49$ (90\% CI: 0.58-3.81).

Conclusions-These results support the original hypothesis. Further studies are needed to confirm or refute the findings here and to clarify the types of hydrocarbon involved.
\end{abstract}

(Occup Environ Med 1994;51:631-637)

Keywords: chronic pancreatitis, hydrocarbon exposure, epidemiology

Centre for

Occupational Health

R McNamee

J Hogg

N M Cherry

Department of

Medicine

(Gastroenterology),

J Braganza

P Rose

Department of Public

Health and

Epidemiology,

University of

Manchester

I Leck (emeritus)

Correspondence to:

Correspondence to:

Epidemiological and Health

Sciences, Stopford Building

Oxford Road, Manchester

Oxford Ro

Accepted 16 May 1994
A rising trend of idiopathic pancreatitis has been noted in England, ${ }^{1}$ which together with other observations, suggests that environmental exposure to chemicals (xenobiotics) may be aetiologically relevant. ${ }^{23}$ When a pharmacokinetic study indicated induction of cytochrome $\mathbf{P 4 5 0}$ in most patients, irrespective of alcohol intake, ${ }^{4}$ Braganza et al undertook a preliminary occupational study. ${ }^{5}$ Nineteen consecutive patients with recurrent pancreatitis were assessed, including seven exalcoholics, and 12 with idiopathic disease. Regular exposure to diesel exhaust fumes or degreasing agents and solvents was found for all patients and several of them had noted a relation between periods away from work and absence of symptoms.
As a consequence of these findings, the present study was set up in 1988 to test the hypothesis that exposure to occupational hydrocarbons is a risk factor for chronic pancreatitis. Since chronic pancreatitis is a rare disease (with an annual incidence of between 1 and 10/100 000 in European countries ${ }^{6}$ ) the case-referent method was considered appropriate. At that time alcohol was accepted as a major risk factor for chronic pancreatitis, ${ }^{6-8}$ cigarette smoking was suspected of being independently associated, and there was some evidence that low levels of dietary antioxidants may play a part. ${ }^{910}$ Information on these potential confounders was therefore sought as well as occupational exposure.

\section{Patients and methods}

IDENTIFICATION OF CASES

Potential cases of chronic pancreatitis were identified retrospectively in three steps (fig). Firstly, the Korner episode system, which records details of all hospital inpatient episodes in England and Wales, was used to identify all inpatients in seven health districts in Greater Manchester (hereafter referred to as the study area), who were discharged alive to an address in the study area during the five year period, 1 April 1985 to 31 March 1990, and whose stay in hospital was given any ICD 577 code (diseases of the pancreas).

In the second step, the hospital notes of these patients were inspected for evidence that the standard diagnostic criteria for chronic pancreatitis had been fulfilled. ${ }^{7}$ These are: either typical histological changes, pancreatic calculi, advanced change pancreatitis by endoscopic pancreatography, or unequivocally reduced exocrine secretory capacity (< mean-3SD); otherwise at least two of the following-moderate change pancreatitis by endoscopic pancreatology, moderately impaired exocrine secretory capacity (between mean-3SD and mean-2SD), and intrapancreatic retention cysts shown by computed tomography. Those who first satisfied the diagnostic criteria between 1 April 1983 and 31 March 1990 were considered further if they were aged $18+$ and had an address within the study area at diagnosis, and if their illness was not considered to be secondary to pancreatic cancer, cystic fibrosis, or abdominal trauma, and there was no record of death in their notes.

Finally, efforts were made to contact these patients, after permission had been obtained from their hospital physicians and general medical practitioners. Only those patients 


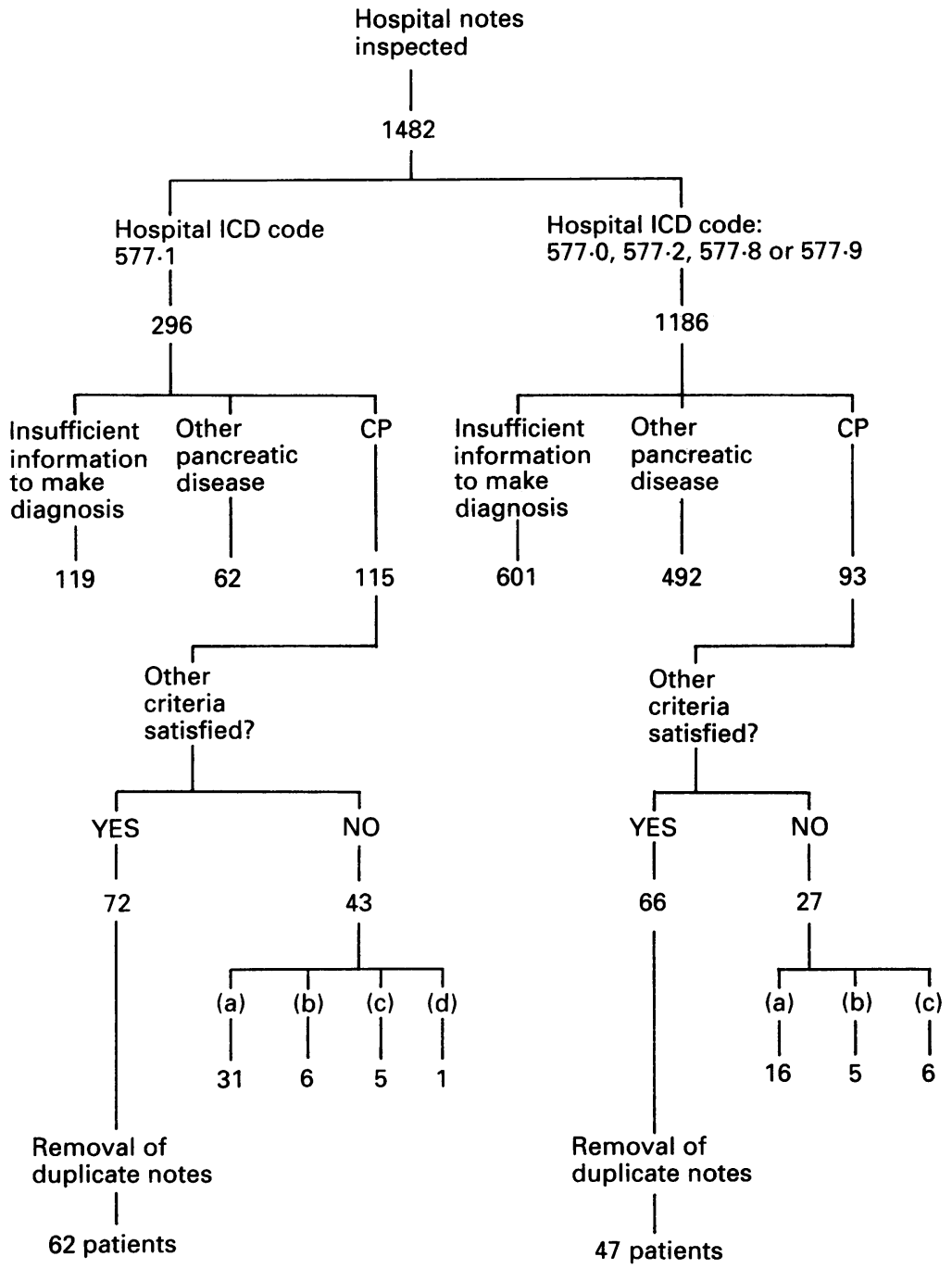

Selection of cases:

ICD codes (9th revision):

$557 \cdot 0$ Acute pancreatitis $(n=1082)$

$577 \cdot 1$ Chronic pancreatitis $(n=296)$;

$577 \cdot 2$ Cysts and pseudocyst of pancreas $(n=25)$,

577.8 Other disease of pancreas $(n=38)$;

577.9 Disease of pancreas-unspecified $(n=41)$

(a) Time of diagnosis outside time frame; (b) residence at diagnosis outside study area;

(c) chronic pancreatitis secondary to pancreatic cancer, trauma, or cystic fibrosis;

(d) deceased. general practitioner, or if the subject had died or could not be contacted, they were replaced by another, chosen in the same way as the original, from the Family Health Services Authority list.

\section{INTERVIEWS}

Structured interviews to ascertain occupational exposure and potential confounding variables were carried out in the homes of cases and referents during the period April 1989 to March 1992. As the interviewer was also involved in identifying study subjects, it was not possible for interviews to be conducted blind to disease state.

\section{ASSESSMENT OF EXPOSURE}

A list of jobs thought to involve potential exposure to hydrocarbons was drawn up at the beginning of the study. At interview all jobs held since leaving school were recorded and, where one of those on the list was reported, there followed a further questionnaire on workplace ventilation, respiratory protection, and work practices.

Copies of the occupational questionnaire were sent to each of two occupational hygienists and two occupational physicians who were not informed about the state of the subject. These assessors independently rated each job both for likelihood (definitely no, probably no, probably yes, definitely yes) and intensity (low, medium, high) of exposure to each of 12 groups of chemicals. In guidelines for rating exposure, it was suggested that the labels low, medium, and high be thought of as $<30 \%$, $30 \%-80 \%$ and $>80 \%$ of occupational exposure limits where these existed.

A method for calculating a cumulative hydrocarbon exposure (CHE) score was devised independently of the data and is shown in the appendix. Only ratings of probable or definite exposure of a medium or high intensity were considered further, with all such medium and high intensity ratings given scores of 1 and 2 respectively. These scores were combined together in three consecutive steps: across chemical groups, across assessors, and across jobs. In step one, a single score for each combination of job and assessor (the assessor job scores) was calculated by adding together the chemical specific scores. At step two, a single score for each job was found by taking the median of the four individual assessor scores (the median job score). Finally, a CHE score up to the date of diagnosis in cases (or the same date for their age matched referents) was found as a weighted sum of the scores for each job, the weights reflecting time spent in the jobs.

Three categories of lifetime exposure were defined from the CHE score: 0 (zero), $0<$ CHE <10 (low), and CHE $\geqslant 10$ (high), and were used to examine the relation between exposure and risk.

Cumulative exposure scores (CEs) were also compiled for each of the 12 chemical groups separately by combining ratings across assessors and jobs only (see appendix).

Information on exposures outside the work 
environment-for example, domestic exposure, hobbies - was also collected, but as the intensity was never more than the equivalent of low occupational exposures, it was not considered further.

\section{ASSESSMENT OF POTENTIAL CONFOUNDERS}

At interview, subjects were questioned about alcohol, cigarettes, and dietary intake, both at the time of interview and two years before the estimated time of first symptoms. Questions about current consumption of alcohol and cigarettes were based on those used in the United Kingdom Government General Household Survey. ${ }^{11}$ A weekly food frequency questionnaire derived from previous work was also completed. ${ }^{910}$ To measure presymptom intakes of all variables, subjects were first questioned about present consumption and then asked how their consumption $x$ years ago differed (where $x$ for each case and the two matching referents was two years earlier than the dates of onset of symptoms in the case). Only presymptom intakes are used in this study.

For the main analysis, weekly alcohol consumption was expressed as a multiple of the recommended safe upper limits for men (21 standard units) and women (14 standard units). ${ }^{12}$ Cigarette consumption before symptoms was classified as "ever" or "never". The dietician, who was unaware of subjects' disease state, derived dietary intakes of antioxidants from questionnaire responses. These data were then expressed as percentages of recommended daily intakes.

To assess social class, all job titles were coded to the Office of Population Censuses and Surveys 1990 standard occupational classification $^{13}$ from which the United Kingdom standard social class groups (I: professional to $\mathrm{V}$ : unskilled) were derived. Where a subject held jobs with different social classifications, he or she was allocated to the class in which they spent most time. Three groups were formed for the main analysis: social classes I + II, III, and IV + V. A second measure of class was based on age at which full time education ended: at the minimum school leaving age, up to two years after the minimum, and later.

\section{STATISTICAL ANALYSIS AND POWER}

Conditional logistic regression techniques were used to estimate odds ratios (ORs) and to control for confounding. Where appropriate, $90 \%$ confidence intervals $(90 \% \mathrm{CIs})$ were calculated, which may also be interpreted as one tailed tests $(a=0.05)$ of the hypothesis under study. To test for trend with level of exposure, a regression coefficient was estimated from the data on those with exposure scores $>0$; this coefficient divided by its standard error was used to provide an (approximate) one tailed test.

Under the assumption that $15 \%$ of the referents would be exposed to hydrocarbons, it was estimated that the study would have $70 \%$ power to detect an increased risk corresponding to an OR of 2 and $90 \%$ power for an OR of
$2 \cdot 5$, with a one-tailed test with a significance level of $5 \%$. Analyses of the relations between individual chemical groups and risk would be expected to have low power.

\section{Results}

ASCERTAINMENT OF CASES AND REFERENTS

The Korner episode system search yielded 1482 hospital case notes of which 296 had been coded as ICD $577 \cdot 1$ (chronic pancreatitis). On inspection, only 72 of these satisfied all the criteria, which gave 62 patients after elimination of duplications (fig). A review of notes with other ICD 577 codes yielded a further 66 eligible notes, corresponding to 47 patients. Of the total of 109 patients thus identified, four had died before the study started, one declined to take part, and two could not be contacted, leaving 102 cases.

Two hundred and four people were initially chosen from Family Health Services Authority files to serve as referents, but only $129(63 \%)$ of these were included in the study. The main reasons for non-participation were: general practitioner refused permission $(7 \cdot 4 \%)$, subject refused $(16 \cdot 7 \%)$, and no response from subject $(11 \cdot 8 \%)$. Where nonresponse could be investigated-that is, in all districts except that where the Family Health Services Authority made the initial contactthe overwhelming reason was that the subject no longer lived at the address held by the Family Health Services Authority or general practitioner. To replace the 75 refusals and non-responders, a further 110 names had to be found from Family Health Services Authority lists, giving a total of 314 names.

The median age of cases at the time of diagnosis was 44 years (range 19-81) and 77 $(75 \%)$ of them were men. Seventy per cent had a presymptom weekly alcohol intake above the safe level compared with $45 \%$ among referents (table 1). Cases left school at an earlier age than referents and were more likely to belong to social class IV or V; a larger proportion of them had a diet that was deficient in ascorbic acid or selenium and they were more likely to have smoked.

\section{OCCUPATIONAL EXPOSURES}

Table 2 summarises the occupational exposure profiles of cases and referents where a subject is classified as having had a particular exposure if they have a CE score $>0$ (individual chemical groups) or a CHE score $>0$ (any chemical group). The most common exposure was to paint solvents which includes paint, paint thinners, varnishes, etc (59 subjects), followed by diesel exhaust fumes (33 subjects), paraffin, kerosine, petrol, or diesel (28 subjects), and chlorinated solvents (21 subjects). Fifty five percent $(n=56)$ of cases and $40 \%(n=81)$ of referents had a CHE score $>0$, which is considerably more exposure in both groups than anticipated.

The crude (unadjusted) OR, which contrasts those with a positive CHE score and those with a zero score was $2 \cdot 21 \quad(90 \% \mathrm{CI}$ : $1 \cdot 38-3.53$ ) (table 3); the OR for the low 
Table 1 Alcohol, cigarette, and dietary antioxidant intake, and social class of cases and referents

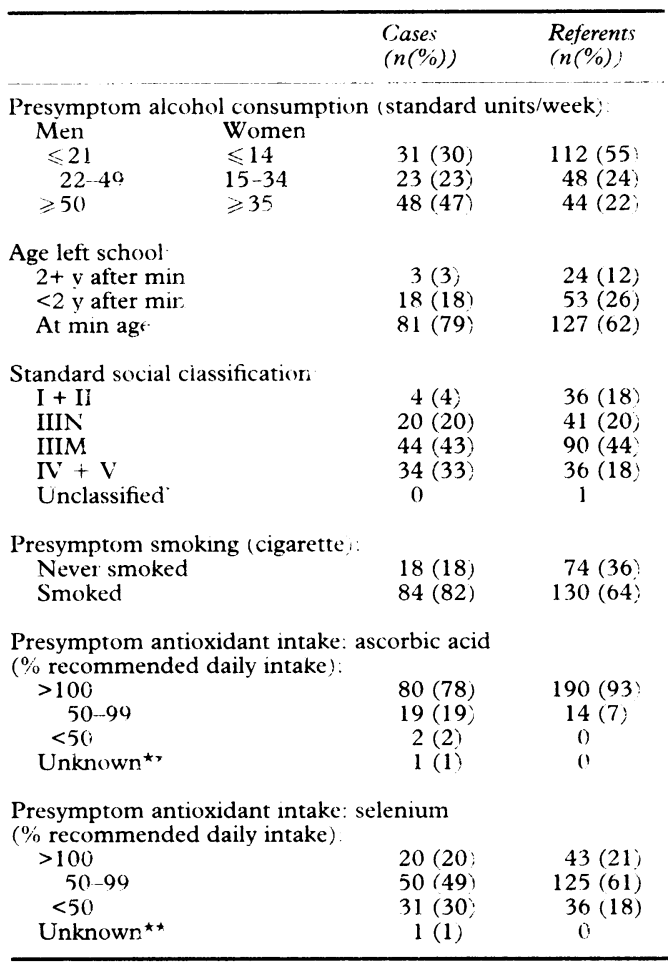

^Never worked outside the home

$\star \star$ Dietarv information incomplet

exposure score category was $1.61(90 \% \mathrm{CI}$ : $0.93-2.77)$ and for the high $3.61(90 \% \mathrm{CI}$ : 1.91-6.82). Among those with scores $>0$, a trend showing increasing ORs with increasing score was significant at the $5 \%$ level $(p=$ $0 \cdot 05)$.

The risk of chronic pancreatitis showed a strong (roughly exponential) relation with presymptom alcohol consumption. Alcohol consumption was also positively correlated with hydrocarbon exposure in the referent group. Together these two associations indicate confounding by alcohol consumption. ${ }^{1+}$ To control confounding, alcohol consumption was included in a conditional logistic regression model, as were the two measures of social class and smoking state. Those antioxidant measures that showed independent associations with risk of chronic pancreatitisascorbic acid and selenium-were aiso included. Four subjects are excluded from this analysis because of missing information.

Table 2 Cases and referents with occupational exposure scores $>0$

\begin{tabular}{lrcr}
\hline Type of exposure & $\begin{array}{c}\text { Cases } \\
(n(\%),\end{array}$ & $\begin{array}{c}\text { Referents } \\
(n(\%))\end{array}$ & $\begin{array}{r}\text { Total } \\
(n(\%))\end{array}$ \\
\hline Dyes & $5(5)$ & $4(2)$ & $9(3)$ \\
Petrol exhaust fumes & $8(8$, & $10(5)$ & $18(6)$ \\
Diesel exhaust fumes & $15(15)$ & $18(9)$ & $33(11)$ \\
Glues, adhesives & $5(5)$ & $6(3)$ & $11(4)$ \\
Weedkillers, pesticides & $2(2)$ & $1(0 \cdot 5)$ & $3(1)$ \\
Oil mists & $4(4)$ & $10(5)$ & $14(5)$ \\
Paint solvents & $23(23)$ & $36(18)$ & $59(19)$ \\
Paraffin, kerosine. & $15(15)$ & $13(6)$ & $28(9)$ \\
$\quad$ petrol, diesel & $2(2)$ & $2(2)$ & $4(1)$ \\
Disinfectants & $10(10)$ & $11(5)$ & $21(7)$ \\
Chlorinated solvents & $3(3)$ & $6(3)$ & $9(3)$ \\
Printing inks & $2(2)$ & $2(1)$ & $4(1)$ \\
Rubber fume & $56(55)$ & $81(40)$ & $137(45)$ \\
Any of these & & & \\
\hline
\end{tabular}

Table 3 Crude and adjusted ${ }^{\star}$ ORs for hydrocarbor exposure as measured by $\mathrm{CHE}$ score

CHE Cases Referents Crude $O R \quad$ Adjusted $O R$
score $(n(\%))(n(\%)) \quad(90 \% C I) \quad\left(90^{\circ} \% C I\right.$

$0446(45) \quad 124(60) \quad 1.00 \quad 1.00$

Low 32 (31) $60(29) \quad 1.61(0.93-2.77) \quad 1.20(0.61-2.35$ High $24(24) \quad 21(10) \quad 3.61(1.91-6.82) \quad 2.67(1.22-5.87$ $>0 \quad 56(55) \quad 56(55) \quad 2.21(1.38-3.53) \quad 1.64(0.92-2.93$

^Adjusted for social class (two measures), alcohol, cigarette consumption, and dietary intakes of selenium and ascorbic acide before symptoms. Four subjects were excluded from adjusted analyses--three because one case did not complete the dietary assessment and so she and her matching referent could not be used, and one (referent) hecause she could not be classified by social class.

Table 3 also shows the adjusted ORs for low and high CHE categories given by this analysis. Both the ORs have been reduced but the adjusted OR of 2.67 for those with a high. score is still significantly increased $(90 \% \mathrm{CI}$ : $1 \cdot 22-5 \cdot 87)$. The test for trend among those with scores $>0$ now gave $p=0.09$.

The relations between $\mathrm{CHE}$ and risk of chronic pancreatitis were examined separately for those who drank less than harmful levels of alcohol (as defined above) and those who drank more than this. To reduce confounding of the associations between risk and CHE by level of consumption within each of these two categories of alcohol, the continuous variablc measuring alcohol intake was included as a covariate in these analyses. In the low alcohol group, the ORs for none, low, and higt hydrocarbon scores were $1.00,1.75(90 \% \mathrm{CI}$ $0 \cdot 77-3 \cdot 98)$, and $4 \cdot 20(90 \% \mathrm{CI}: 1 \cdot 41-12 \cdot 57)$, In the high alcohol group, the corresponding ORs calculated relative to the no exposure, low alcohol group were $3.80 \quad(90 \% \quad \mathrm{CI}$ : $0 \cdot 97-14 \cdot 86), 2 \cdot 29(90 \% \mathrm{CI}: 0 \cdot 49-10 \cdot 74)$, and $5 \cdot 51(90 \% \mathrm{CI}: 1 \cdot 11-27 \cdot 36)$. The association with hydrocarbon exposure seems weaker for alcoholic compared with non-alcoholic chronic pancreatitis, but the numbers are ton small to allow firm conclusions for the alcoholic group.

Table 4 shows the associations between risk of chronic pancreatitis and the four chemica!

Table 4 Crude and adjusted ORs for exposure to selected chemicals

\begin{tabular}{|c|c|c|c|c|}
\hline $\begin{array}{l}C E \\
\text { Score }\end{array}$ & $\begin{array}{l}\text { Cases } \\
(n(\%))\end{array}$ & $\begin{array}{l}\text { Referents } \\
(n(\%))\end{array}$ & $\begin{array}{l}\text { Crude } \\
\text { OR }\end{array}$ & $\begin{array}{l}\text { Adjusteà } \\
O R\left(90^{\circ}, C I\right.\end{array}$ \\
\hline \multicolumn{5}{|c|}{ Paint solvents. } \\
\hline 0 & 79 & 168 & $1 \cdot 00$ & $1 \cdot 00$ \\
\hline Low $^{\star}$ & 14 & 25 & $1 \cdot 22$ & $1.02(0.43-2.44$ \\
\hline High * & 9 & $1:$ & 1.79 & $0.87(0.31-2.52$ \\
\hline$>0$ & 23 & 36 & $1 \cdot 41$ & $0.96(0.48 \cdot 1.93$ \\
\hline \multicolumn{5}{|c|}{ Diesel exhaust fumes: } \\
\hline 0 & 87 & 180 & $1 \cdot 00$ & $1 \cdot 00$ \\
\hline Low* & 10 & 12 & 1.96 & $1.90(0.63-6.09)$ \\
\hline $\mathrm{High}^{\star}$ & 5 & $i$ & $2 \cdot 08$ & $4.18(1.09-16.06)$ \\
\hline$>0$ & 15 & 18 & $2 \cdot 04$ & $2.66(1.05-6.73$ \\
\hline \multicolumn{5}{|c|}{ Paraffin, kerosine, petrol, or diesel: } \\
\hline 0 & 87 & 191 & 1.00 & 1.00 \\
\hline Low $^{\star}$ & 11 & 8 & 3.09 & $2.70(0.99 \cdot 7.31)$ \\
\hline $\mathrm{High}^{\star}$ & 4 & 5 & $1 \cdot 86$ & $0.92(0.26-3.30$ \\
\hline$>0$ & 15 & 13 & $2 \cdot 62$ & $1 \cdot 82(0 \cdot 8()-4 \cdot 11)$ \\
\hline \multicolumn{5}{|c|}{ Chlorinated solvents' } \\
\hline 0 & 92 & 193 & $1 \cdot 00$ & $1 \cdot 00$ \\
\hline Low ${ }^{\star}$ & 7 & 9 & 1.62 & $0.96(0.30-3.04)$ \\
\hline High $^{\star}$ & 3 & 2. & $3 \cdot 1 \%$ & $4.41(0.69-28.19$ \\
\hline$>0$ & 10 & 11 & $1 \cdot 88$ & $1.49(0.58-3.81)$ \\
\hline
\end{tabular}

${ }^{\star}$ Low: $0<$ score $<5$. High: score $\geqslant 5$

Adjustments and exclusions as explained in table 
groups for each of which there were at least 20 exposed subjects. The ORs are adjusted for alcohol, smoking, dietary and social class variables as before, but not other occupational exposures. Because of the small number of people with scores $>10$, a cut off point of 5 was used to define high exposure for these variables. Although there is a suggestion of a relation between paint solvents and risk in the crude data, this disappears after adjustment for confounding. The adjusted OR for diesel exhaust exposure is significant at the $5 \%$ level, $\mathrm{OR}=2 \cdot 66,(90 \% \mathrm{CI}: 1 \cdot 05-6 \cdot 73)$, and there is some suggestion of a dose-response relation. The ORs for paraffin, kerosine, petrol, or diesel (1.82), and for chlorinated solvents $(1 \cdot 49)$ are not significantly increased.

\section{Discussion}

A positive association has been found between a high $(\geqslant 10)$ CHE score and risk of chronic pancreatitis. Aspects of the study methodology that might be judged logically to have contributed to a false positive association, and the implications of the results, are considered separately.

\section{METHODOLOGY}

Undoubtedly some cases of chronic pancreatitis in the study area have been omitted because the required diagnostic procedures were not carried out, but as these exclusions were not related to exposure, this is not a source of bias. Similarly, the restriction to cases still alive at the time of the study is unimportant unless exposure is related to survival.

Ideally, the referents should have been chosen from those people resident in the study area at the time of diagnosis of the cases (1 April 1983 to 31 March 1990). It was not possible to sample this population retrospectively; instead referents were chosen from residents at the time of the study. This could lead to bias if the exposure profile of the population has changed over time. Information on the previous residence of all study subjects was available and showed that six referents had lived outside the study area at the time of diagnosis of their matching case. A reanalysis omitting these six gave very similar results (OR for high CHE score $=2 \cdot 59$ ).

The response rate $(63 \%)$ among the 204 people originally chosen as referents was somewhat low, and non-participants were replaced. This could lead to a false positive result if participating referents had less exposure than non-participants. This could occur if, for example, participation was related to social class. As almost the entire population is registered with a Family Health Services Authorities in England and Wales, the social class of referents should be broadly similar to that of the entire population of the study area. To check this, a comparison of the social classes of the referents with those of age, sex, and area specific figures from the 1981 census of Great Britain ${ }^{15}$ was carried out; this showed that the referents in the study were slightly more likely to belong to the manual classes
$(62 \% v 57 \%)$. Thus it seems unlikely that population exposure has been underestimated in this referent group.

Estimation of past exposure to hydrocarbons was carried out in three stages: interview, assessment of interview material by a panel, and construction of an exposure score from these assessments. Some misclassification with respect to exposure is inevitable when it is assessed retrospectively, but provided this operates equally in both case and referent groups, it should not produce false positive associations. ${ }^{14}$ Study subjects were not told of the study hypothesis, but as the interviewer was aware of the group to which subjects belonged, the possibility of information bias must be acknowledged. The translation of job descriptions into exposures, however, was done by a team who were unaware whether the subject was a case or a referent. Also reassuring was the interviewer's opinion, on completion of all interviews, that the job profiles of cases and referents seemed to be similar.

The CHE measure of exposure used here was just one of several possible approaches to summarising the exposure data but, importantly, it was the one chosen in advance. In retrospect, this method of classifying jobs as "exposed" might be said to have erred on the side of sensitivity at the expense of specificity: to receive a score $>0$, only two of the four assessors needed to rate the job as exposed. In fact, had a stricter criterion-that three of the four were needed-been used, the strength of the association with risk would have increased. (Adjusted ORs for low CHE scores would then be $1 \cdot 32(90 \% \mathrm{CI}: 0 \cdot 62-2 \cdot 80)$ and for high scores $3 \cdot 17(90 \%$ CI: 1·41-7·13)).

Furthermore, the CHE measure followed a "broad-brush" approach in terms of (a) the timing of exposure ("ever" before diagnosis); (b) assumptions about the respective effects of intensity and duration of exposure, and (c) type of hydrocarbons (any of the groups in table 2). This approach seems warranted in the absence of a more specific hypothesis. Retrospective analyses to clarify the roles of intensity, duration, and timing in relation to disease presentation were carried out and, although inconclusive, were consistent with the view that exposure had a cumulative effect.

To allow for confounding, the interview schedule included questions on all known or postulated risk factors for chronic pancreatitis, and these were included as covariates in a regression model. In general, adjustment for confounding effects by statistical analysis does not remove the problem in its entirety, as error in the measurement of confounders results in residual confounding after adjustment. ${ }^{16}$ In fact, after adjustment for other variables the relations between social class and chronic pancreatitis, and smoking and chronic pancreatitis were not significant. Inclusion of these variables together with the dietary measures made little difference to the estimates of ORs for hydrocarbon exposure.

Alcohol consumption is a particularly 
strong risk factor for chronic pancreatitis and therefore the methods for measurement and analytical control of this variable are critically important. It was the interviewer's impression that subjects were quite willing to admit to heavy drinking in the past. This, and the heavy consumption of alcohol reported by both cases and referents, suggests that previous consumption may be less prone to the under reporting that is said to occur for current habits. In the statistical analysis efforts were made to model the relation between alcohol and risk of chronic pancreatitis as accurately as possible. The inclusion of alcohol as a continuous variable (with the attendant assumption of an exponential relation) was considered to be the most accurate approach. It also reduced the association between the CHE score and risk of chronic pancreatitis more than other methods-for example, with categories of alcohol. It seems unlikely therefore that the association can simply be explained by the confounding effects of alcohol.

\section{IMPLICATIONS}

If the OR of 2.67 associated with a high exposure score is correct, then with calculations based on the attributable risk, one can estimate that exposure at this level played a part in 15 cases (15\% of the total) in this urban study population. The industries or jobs of those placed in the high exposure group were varied, but with metal production or working, transport, and motor repair being the main sectors. The SOC code 540 , motor mechanics and auto engineers, (who can be exposed to paints as well as petrochemicals) was a particularly common code in this group, but it should be noted that there is not a one to one correspondence between job types and exposure.

In the subsidiary analyses, which had limited statistical power, the greatest risk seemed to be associated with diesel exhaust fumes. It is not evident why this product should be a particular hazard as its hydrocarbon content is fairly low, but it may contain other toxic substances. If hydrocarbons alone play a part in chronic pancreatitis, then those chemical groups that are almost purely hydrocarbon (groups 7 and 8) might be expected to show the strongest associations. Surprisingly, no relation was found with paint solvents.

It has been hypothesised that chronic pancreatitis is a product of heightened but unmitigated oxidative detoxification reactions mediated by cytochromes P450.2317 Under this model, aetiologically relevant chemicals would be inducers of P450 and undergo metabolic activation. Damage would only occur if the supply of antioxidants were insufficient to meet the increased demand from reactive oxygen or xenobiotic metabolites. ${ }^{1017}$ In this study, a relation between chronic pancreatitis and antioxidant intake was found. Consideration of this and the interactions between antioxidant intake and occupational exposures in determining risk of chronic pancreatitis will be examined elsewhere.
Chronic pancreatitis is a rare disease but a disabling one; if occupational exposure to organic chemicals is indeed a risk factor, as suggested by our study, then identification of the most heavily implicated chemicals, and reduction of these exposures, must be a priority. If further studies are set up to confirm (or refute) the main results here, then it would be desirable to put particular emphasis on the valid differentiation between different types of chemicals containing hydrocarbons.

We thank the North Western Regional Health Authority who funded this study, and G Fletcher, W R Lee, M Molyneux, and $D$ Turner who carried out the occupational assessments. We also thank the Medical and Ethics Committees and Family Health Services Authorities of the seven Health Districts in Health Services Authorities of the seven Health Districts in Greater Manchester (North, Central, and South Manchester, Salford, Stockport, Tameside, and Trafford) and
themselves who gave their consent to the study.

\section{Appendix: illustration of derivation of exposure scores}

(A) CUMULATIVE HYDROCARBON EXPOSURE (CHE) SCORE TO DIAGNOSIS

Hypothetical job with scores $>0$ for chemical groups 3,8 , and 9 only

\begin{tabular}{lllll}
\hline & \multicolumn{2}{l}{ Chemical } & group & Assessor's \\
\cline { 2 - 5 } Assessor & 3 & 8 & 9 & job score \\
\hline 1 & 0 & $\mathrm{M}$ & $\mathrm{M}$ & 2 \\
2 & 0 & 0 & $\mathrm{M}$ & 1 \\
3 & 0 & $\mathrm{M}$ & 0 & 1 \\
4 & $\mathrm{M}$ & $\mathrm{H}$ & $\mathrm{M}$ & 4 \\
Median & - & - & - & 1.5 \\
\hline
\end{tabular}

Cumulative hydrocarbon exposure score to diagnosis = $\Sigma_{\mathrm{J}}$ (median intensity for group $3 \times$ years in job $\times$ hours per week $/ 40)$.

$\mathrm{j}=$ all jobs to diagnosis.

(B) CUMULATIVE EXPOSURE (CE) SCORE UP TO DIAGNOSIS FOR EACH CHEMICAL GROUP SEPARATELY

\begin{tabular}{llll}
\hline & \multicolumn{3}{l}{ Chemical } \\
\cline { 2 - 4 } Aroup & \\
\cline { 2 - 4 } Assessor & 3 & 8 & 9 \\
\hline 1 & 0 & $\mathrm{M}$ & $\mathrm{M}$ \\
2 & 0 & 0 & $\mathrm{M}$ \\
3 & 0 & $\mathrm{M}$ & 0 \\
4 & $\mathrm{M}$ & $\mathrm{H}$ & $\mathrm{M}$ \\
Median & 0 & 1 & 1 \\
\hline
\end{tabular}

Cumulative exposure to diagnosis for chemical group 3, say $\Sigma_{\mathrm{J}}$ (median intensity for group $3 \times$ years in job $\times$ hours per week/40)

$\mathrm{j}=$ all jobs to diagnosis.

1 James O, Agnew JE, Bouchier IAD. Chronic pancreatitis in England: a changing picture? $B M F 1974 ; 2: 34-8$.

Braganza JM. Hypothesis. Pancreatic disease: a casualty of hepatic "detoxification"? Lancet 1983;ii: 1000-3.

3 Braganza JM. Chronic pancreatitis. In: International teaching sessions of the golden jubilee. Meeting of the British Society of sessions of the golden jubilee. Meeting of the British

4 Acheson DWK, Rose P, Houston JB, Braganza JM. Induction of cytomchromes P-450 in pancreatic disease: Induction of cytomchromes $\mathrm{P}-450$ in pancreatic disease: consequence, coinci

5 Braganza JM, Jolley JE, Lee WR. Occupational chemicals and pancreatitis: a link? Int $\mathcal{f}$ Pancreatol 1986;1:9-19.

6 Worning H. Chronic pancreatitis: pathogenesis, natural history and conservative treatment. Clin Gastroenterol, 1984;13:871-94.

7 Braganza JM. The pancreas. In: Pounder RE, ed. Recent advances in gastroenterology. Vol 6. London: Churchill Livingstone, 1986;251-80. 
8 Yen S, Hsieh C, MacMahon B. Consumption of alcohol and tobacco and other risk factors for pancreatitis. $A m$ Epidemiol 1982;116:407-14

9 Rose P, Fraine E, Hunt LP, Acheson DWK, Braganza JM. Dietary anti-oxidants and chronic pancreatitis. Human Nutrition: Clinical Nutrition 1986;40c:151-64.

10 Uden S, Acheson DWK, Reeves J, Worthington HV, Hunt LP, Brown S, et al. Antioxidants, enzyme induction and LP, Brown S, et al. Antioxidants, enzyme induction

11 Office of Population Censuses and Surveys. General household survey 1984. London: HMSO, 1986.

12 Royal College of General Practitioners. Alcohol-a bal- anced view. Report from general practice 1986:24. 13 Office of Population Censuses and Surveys. Standard occupational classification. London: HMSO, 1990.

14 Rothman K. Modern epidemiology. Boston: Little, Brown 1986.

15 Office of Population Censuses and Surveys. Census 1981 county report, Greater Manchester, part 2. London: county report,

16 Greenland $S$. The effect of misclassification in the presence of covariates. Am F Epidemiol 1980;112:564-9.

17 Braganza JM, ed. The pathogenesis of pancreatitis. Manchester: Manchester University Press, 1991.

\section{Vancouver style}

All manuscripts submitted to Occup Environ Med should conform to the uniform requirements for manuscripts submitted to biomedical journals (known as the Vancouver style.)

Occup Environ Med, together with many other international biomedical journals, has agreed to accept articles prepared in accordance with the Vancouver style. The style (described in full in the $B M \mathcal{F}, 24$ February $1979, p 532$ ) is intended to standardise requirements for authors.

References should be numbered consecutively in the order in which they are first mentioned in the text by Arabic numerals above the line on each occasion the reference is cited (Manson ${ }^{1}$ confirmed other reports $^{2-5}$. . .). In future references to papers submitted to Occup Environ Med should include: the names of all authors if there are seven or less or, if there are more, the first six followed by et al; the title of journal articles or book chapters; the titles of journals abbreviated according to the style of Index Medicus; and the first and final page numbers of the article or chapter. Titles not in Index Medicus should be given in full.

Examples of common forms of references are:

1 International Steering Committee of Medical Editors, Uniform requirements for manuscripts submitted to biomedical journals. Br Med ₹ 1979;1:532-5.

2 Soter NA, Wasserman SI, Austen KF. Cold urticaria: release into the circulation of histamine and eosinophil chemotactic factor of anaphylaxis during cold phil chemotactic factor of anaphylaxis durit

3 Weinstein Weinstein L, Swartz MN. Pathogenic properties of
invading micro-organisms. In: Sodeman WA Jr, Sodeman WA, eds. Pathologic physiology, mechanism of disease. Philadelphia: W B Saunders, 1974:457-72. 